This item was submitted to Loughborough's Institutional Repository (https://dspace.lboro.ac.uk/) by the author and is made available under the following Creative Commons Licence conditions.

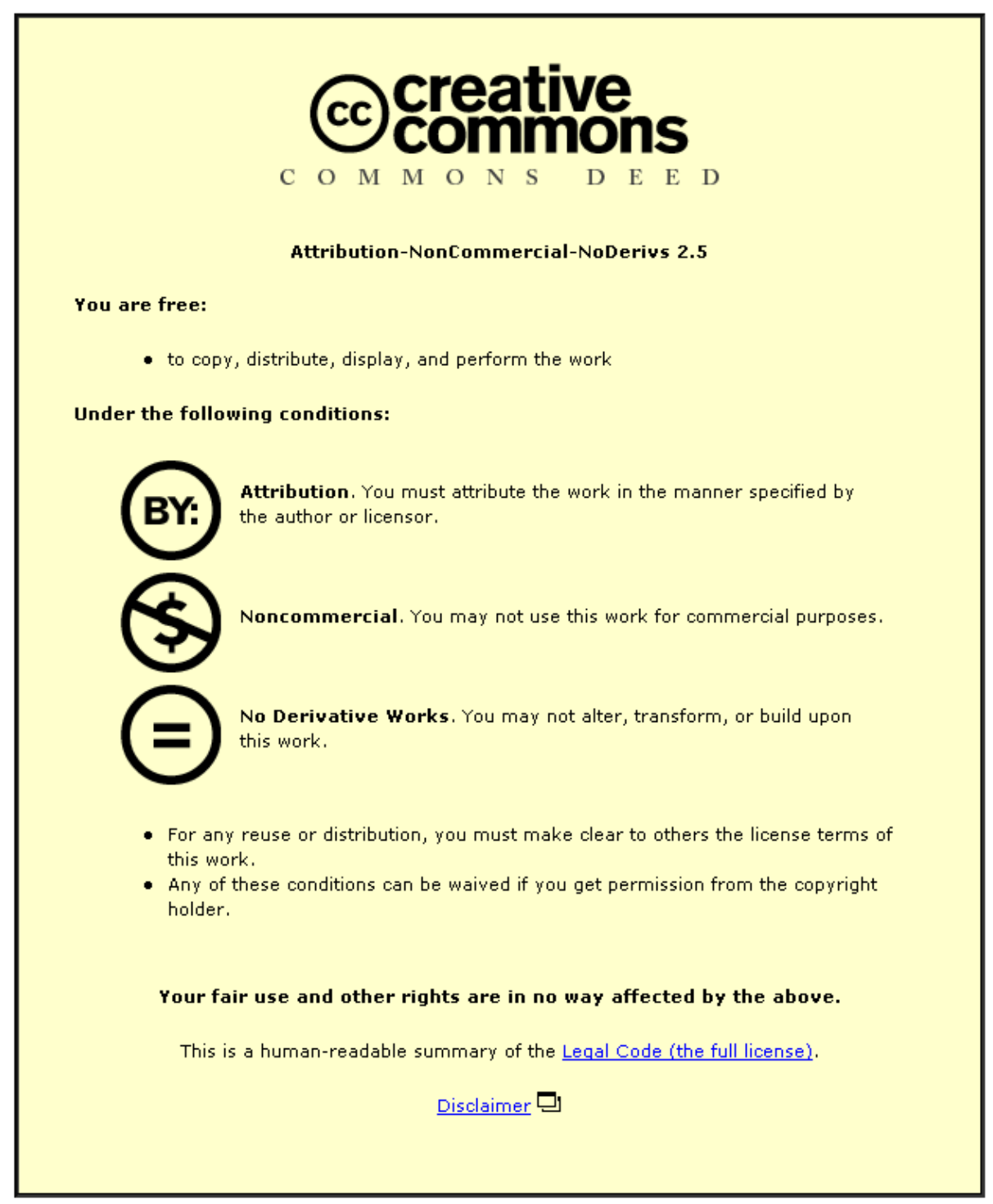

For the full text of this licence, please go to: http://creativecommons.org/licenses/by-nc-nd/2.5/ 


\title{
The biomechanics of twisting somersaults. Part I: Rigid body motions
}

\author{
M.R. YEADON* \\ Sports Biomechanics Laboratory, Department of Physical Education and Sports Science, \\ Loughborough University, Ashby Road, Loughborough LE11 3TU, UK \\ and Human Performance Laboratory, University of Calgary, Canada
}

\begin{abstract}
This series of four papers comprises a theoretical investigation into twisting somersaults. Both simple and complex mathematical models are used to provide an understanding of the mechanics of the production and removal of twist in somersaults. Various twisting techniques are evaluated and a method is developed for the partitioning of an actual performance into contributions from these twisting techniques.

In Part I, analytical solutions for the torque-free rotational motion of a rigid body are derived. It is shown that there are two distinct modes of motion which may be characterized as a twisting somersault and a wobbling somersault. The phenomenon of unstable rotations about the intermediate principal axis is explained in terms of these two modes.
\end{abstract}

Keywords: Twist, somersault, model, simulation, biomechanics.

\section{Introduction}

Twisting somersaults are the main elements of gymnastics, diving, tumbling, trampolining and freestyle skiing. It has been recognized that twisting may be started during the takeoff phase when the athlete is still in contact with the apparatus (Rackham, 1960). It has been proposed that the initiation of such contact twist is achieved by twisting the head, arms and shoulders (Aaron, 1977), the upper body (Batterman, 1974) or the legs (Smith, 1980). The question of the relative merits of such techniques has not been addressed thus far. Eaves (1960) stated that taking twist from the board leads to tilt at the end of a dive. How divers can cope with this problem and enter the water with a straight body position without further twisting needs explanation.

The fact that a cat can twist over when dropped in an inverted position with zero angular momentum demonstrates that it is possible for an animal to produce twist during the aerial phase (McDonald, 1960). When somersault is present, the angular momentum is not zero and there exists the possibility of using this angular momentum to produce an aerial twist. Pike (1980) showed that theoretically a full twist may be introduced into a plain dive by means of asymmetrical arm movements during the aerial phase. Frohlich (1980) observed that in addition to arm movements, other asymmetrical movements of body segments during a somersault will result in an aerial twist. Such techniques need to be identified and the factors governing their effectiveness evaluated.

Nigg (1974) and Hinrichs (1978) have shown that the layout somersault is inherently unstable. With a better understanding of the mechanics of twisting somersaults, it should be possible to see why such instability occurs, how it manifests itself and how an athlete can cope with the instability.

This series of four papers endeavours to answer such questions. A theoretical rather than experimental approach has been taken, since a theoretical study can be well controlled, whereas attempts to change movement patterns in humans may produce more complex changes than intended. Specific 'what if?' questions will be answered using the 11-segment computer simulation model of Yeadon et al. (1990).

\footnotetext{
*Address all correspondence to M. R. Yeadon, Department of Physical Education and Sports Science, Loughborough University, Ashby Road, Loughborough LE1 1 3TU, UK.
} 
While the use of a computer model can provide answers to specific questions, it may not be possible to gain insight into the underlying mechanics and to explain why a certain effect occurs. One way of gaining such mechanical insights is to use a greatly simplified model that still exhibits some of the features of interest. It is then often possible to obtain general analytical solutions that give insight into the behaviour of the system over a range of conditions. This series has made use of a number of such simplified models.

In Part I, a rigid body model of aerial movement is used to describe the general rotational motions of a torque-free body and to obtain relationships between twist, somersault and tilt angles and their rates of change. Once divers and gymnasts have started to twist in a somersault, they often appear to maintain a fixed body configuration. A rigid body may be expected to give a reasonable representation of such phases and to describe how the three orientation angles vary throughout the motion. In the case of a rigid body with an axis of inertial symmetry, the mathematics is greatly simplified and the ratio of twist and somersault rates may be expressed as a function of the tilt angle as shown by Eaves (1969), Eaves (1971) and Frohlich (1979). Unfortunately, these three derivations result in three different functions which all purport to describe the same relationship. These discrepancies are explained and the correct form of the relationship is derived. The general description of rigid body motion is used to explain why rotations are stable about the principal axes corresponding to the greatest and least moments of inertia but unstable about the remaining principal axis. The manner in which this instability manifests itself is described.

In Part II, the relative abilities of arms, chest and whole body movements to produce twist during the contact phase are investigated using simple models. The effect that the introduction of contact twist has on the somersault rate is determined using the rigid body model developed in Part I and mechanisms for controlling the tilt during flight are investigated using the 11-segment simulation model of Yeadon et al. (1990). Part III considers the initiation of twist during the aerial phase by means of asymmetrical movements of the arms, chest and hips. The effect on the somersault rate of initiating aerial twist is determined and methods for stopping the twist are evaluated using the simulation model.

In Part IV, a method is presented for determining the contributions made by contact and aerial twisting techniques in filmed performances of twisting somersaults. Tilt contributions are determined for four competitive movements performed by an elite trampolinist. The implications for coaching are considered and future research studies of twisting somersaults are discussed.

\section{Theory}

The rotational motion of a rigid body under zero net torque is governed by the conservation of angular momentum which may be written as:

$$
\mathbf{h}=\mathbf{I}_{f f} \boldsymbol{\omega}_{f i}
$$

where $\mathbf{I}_{f f}$ is the whole body inertia tensor and $\boldsymbol{\omega}_{f i}$ is the angular velocity of the body relative to the inertial reference frame $i$.

If the reference frame $f$ of the system is chosen to be oriented with the principal axes of the body, then in frame $f$ the inertia tensor $\mathbf{I}_{f f}$ has the diagonal form:

$$
\left(\mathbf{I}_{f f}\right)_{f}=\left[\begin{array}{ccc}
A & 0 & 0 \\
0 & B & 0 \\
0 & 0 & C
\end{array}\right]
$$

where $A, B, C$ are the principal moments of inertia corresponding to the principal axes $\mathbf{f}_{1}, \mathbf{f}_{2}, \mathbf{f}_{3}$.

In frame $f$, the angular momentum is:

$$
(\mathbf{h})_{f}=R_{3}(\psi) R_{2}(\theta) R_{1}(\phi)(\mathbf{h})_{i}
$$

where $R_{1}(\phi), R_{2}(\theta), R_{3}(\psi)$ are rotation matrices corresponding to the somersault angle $\phi$, the tilt angle $\theta$ and the twist angle $\psi$ shown in Fig. 1. Thus if $(\mathbf{h})_{i}=\left[\begin{array}{l}h \\ 0 \\ 0\end{array}\right]$ then $(\mathbf{h})_{f}=\left[\begin{array}{c}h \cos \theta \cos \psi \\ -h \cos \theta \sin \psi \\ h \sin \theta\end{array}\right]$ where the symbol $h$ will now be used to denote a scalar quantity. 


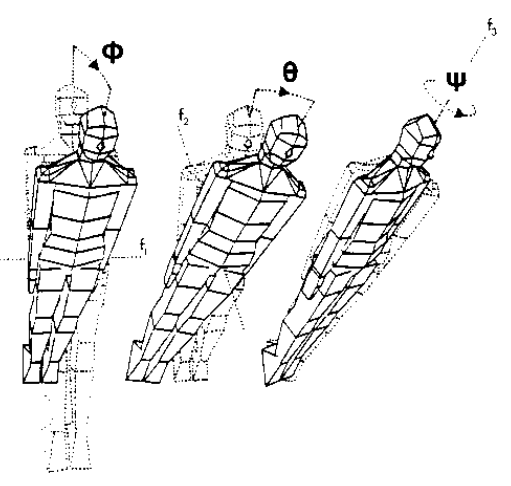

Figure 1: Angles of somersault $(\phi)$, tilt $(\theta)$ and twist $(\psi)$.

In Yeadon (1990a), the angular velocity $\boldsymbol{\omega}_{f i}$ was evaluated in frame $f$ as:

$$
\left(\boldsymbol{\omega}_{f i}\right)_{f}=\left[\begin{array}{ccc}
\cos \theta \cos \psi & \sin \psi & 0 \\
-\cos \theta \sin \psi & \cos \psi & 0 \\
\sin \theta & 0 & 1
\end{array}\right] \cdot\left[\begin{array}{c}
\dot{\phi} \\
\dot{\theta} \\
\dot{\psi}
\end{array}\right]
$$

Equation (1) then takes the form:

$$
\left[\begin{array}{c}
h \cos \theta \cos \psi \\
-h \cos \theta \sin \psi \\
h \sin \theta
\end{array}\right]=\left[\begin{array}{ccc}
A & 0 & 0 \\
0 & B & 0 \\
0 & 0 & C
\end{array}\right] \cdot\left[\begin{array}{c}
\dot{\phi} \cos \theta \cos \psi+\dot{\theta} \sin \psi \\
-\dot{\phi} \cos \theta \sin \psi+\dot{\theta} \cos \psi \\
\dot{\phi} \sin \theta+\dot{\psi}
\end{array}\right]
$$

which gives rise to:

$$
\begin{aligned}
\dot{\phi} & =h\left(A \sin ^{2} \psi+B \cos ^{2} \psi\right) / A B \\
\dot{\theta} & =-h(A-B) \cos \theta \sin \psi \cos \psi / A B \\
\dot{\psi} & =(h / C-\dot{\phi}) \sin \theta
\end{aligned}
$$

The conservation of rotational energy may be derived from equations (2), (3) and (4) using a single integration, or since the absence of an external torque implies that no work is done, the energy equation may be written as:

$$
2 T=A \omega_{1}^{2}+B \omega_{2}^{2}+C \omega_{3}^{2}
$$

where $T$ is the (constant) rotational energy and $\omega_{1}, \omega_{2}, \omega_{3}$ are the components of the angular velocity $\left(\boldsymbol{\omega}_{f i}\right)_{f}$.

Since the angular momentum components in frame $f$ are $h_{1}, h_{2}, h_{3}$ where:

$$
\begin{gathered}
{\left[\begin{array}{l}
h_{1} \\
h_{2} \\
h_{3}
\end{array}\right]=\left[\begin{array}{c}
h \cos \theta \cos \psi \\
-h \cos \theta \sin \psi \\
h \sin \theta
\end{array}\right]} \\
=\left[\begin{array}{lll}
A & 0 & 0 \\
0 & B & 0 \\
0 & 0 & C
\end{array}\right] \cdot\left[\begin{array}{l}
\omega_{1} \\
\omega_{2} \\
\omega_{3}
\end{array}\right]
\end{gathered}
$$

the energy equation may be written as:

$$
2 T=h_{1}^{2} / A+h_{2}^{2} / B+h_{3}^{2} / C
$$

which leads to:

$$
2 T=h^{2} \cos ^{2} \theta \cos ^{2} \psi / A+h^{2} \cos ^{2} \theta \sin ^{2} \psi / B+h^{2} \sin ^{2} \theta / C
$$


If $C$ is the minimum principal moment of inertia, positive constants $c_{\alpha}^{2}$ and $c_{\beta}^{2}$ may be defined as:

$$
\begin{aligned}
& c_{\alpha}^{2}=\left(h^{2} / C-2 T\right) /\left(h^{2} / C-h^{2} / A\right) \\
& c_{\beta}^{2}=\left(h^{2} / C-2 T\right) /\left(h^{2} / C-h^{2} / B\right)
\end{aligned}
$$

so that substitution in equation (5) permits the energy equation to be written in the following two forms:

$$
\begin{aligned}
& \sin ^{2} \psi=\left(1-c_{\alpha}^{2} \sec ^{2} \theta\right) B(A-C) / C(A-B) \\
& \cos ^{2} \psi=\left(c_{\beta}^{2} \sec ^{2} \theta-1\right) A(B-C) / C(A-B)
\end{aligned}
$$

Equations (2) and (8) then give the somersault rate as:

$$
\dot{\phi}=h\left[1 / C-(1 / C-1 / A) c_{\alpha}^{2} \sec ^{2} \theta\right]
$$

while equations (4) and (10) give the twist rate as:

$$
\dot{\psi}=h(1 / C-1 / A) c_{\alpha}^{2} \sec ^{2} \theta \sin \theta
$$

It should be noted that equations (2) through (11) hold for $A \geq B \geq C$ and for $B \geq A \geq C$.

From this point on, let $A \geq B \geq C$. Equation (5) shows that the maximum possible energy for a rigid body with angular momentum $h$ is given by $2 T=h^{2} / C$, corresponding to a pure twist about axis $\mathbf{f}_{3}$, while the minimum energy is given by $2 T=h^{2} / A$, which corresponds to a pure somersault about axis $\mathbf{f}_{1}$.

From equation (6) we may deduce that $0 \leq c_{\alpha}^{2} \leq 1$ and replace $c_{\alpha}^{2}$ by $\cos ^{2} \alpha$ in the above equations knowing that the equation $\cos ^{2} \alpha=c_{\alpha}^{2}$ produces a real value for the angle $\alpha$. When the twist angle $\psi=0$, the tilt angle $\theta$ will be equal to $\alpha$ (equation 8 ).

Following the procedure of Whittaker (1937), equations (3) and (8) give:

$$
\dot{y}^{2}=d^{2}\left(1-y^{2}\right)\left(y^{2}+e\right)
$$

where:

$$
\begin{aligned}
y & =\sin \theta / \sin \alpha \\
d^{2} & =h^{2} \sin ^{2} \alpha(A-C)(B-C) / A B C^{2} \\
e & =\operatorname{cosec}^{2} \alpha C(A-B) / A(B-C)-B(A-C) / A(B-C)
\end{aligned}
$$

Equation (12) may then be integrated to give the time history of the tilt angle $\theta$.

In order to define two general modes of motion, let the angle $\alpha_{0}$ be defined by:

$$
\cos ^{2} \alpha_{0}=A(B-C) / B(A-C)
$$

Equations (6), (7) and (13) give:

$$
\cos ^{2} \alpha=\cos ^{2} \alpha_{0} c_{\beta}^{2}
$$

Equation (8) may now be written in the form:

$$
\cos ^{2} \alpha=\cos ^{2} \theta\left(1-\sin ^{2} \psi \sin ^{2} \alpha_{0}\right)
$$

Comparison with equation (14) shows that $\psi=90^{\circ}$ corresponds to $\cos ^{2} \theta=c_{\beta}^{2}$. There are now two possible cases to consider:

$$
\text { (a) } \quad \alpha>\alpha_{0}
$$

In this case, $\cos ^{2} \alpha<\cos ^{2} \alpha_{0}$ so that there exists a value $\beta$ of $\theta$ satisfying equation (15) when $\psi=90^{\circ}$. In this mode it is possible to reach the quarter twist position.

$$
\alpha<\alpha_{0}
$$

In this case, $\cos ^{2} \alpha>\cos ^{2} \alpha_{0}$ so that equation (14) implies that $c_{\beta}^{2}>1$ and there is no real value of $\theta$ satisfying equation (15) with $\psi=90^{\circ}$. In this mode the quarter twist position is never reached. 


\section{The twisting mode}

If a rod is defined to be an axially symmetric body with principal moments of inertia $A, B, C$ such that $A=B>C$, then $\alpha_{0}=0$ from equation (13) so that $\alpha \geq \alpha_{0}$. For a general rigid body, the motion will be said to be in the rod mode providing $\alpha>\alpha_{0}$. In this case, $0<c_{\beta}^{2}<1$ from equation (14), so that there exists an angle $\beta$ for which $\cos ^{2} \beta=c_{\beta}^{2}$.

From equations (6) and (7) we may now write:

$$
(1 / C-1 / A) \cos ^{2} \alpha=(1 / C-1 / B) \cos ^{2} \beta
$$

This equation relates the values $\alpha$ and $\beta$ of the tilt angle $\theta$ corresponding to the values 0 and $90^{\circ}$ for the twist angle $\psi$.

Equation (12) may be written as:

$$
\begin{aligned}
& \dot{y}^{2}=p^{2}\left(1-y^{2}\right)\left(y^{2}-\left(1-k^{2}\right)\right) \\
& \text { where: } p^{2}=h^{2} \sin ^{2} \alpha(A-C)(B-C) / A B C^{2} \\
& k^{2}=\cot ^{2} \alpha C(A-B) / A(B-C)<1 .
\end{aligned}
$$

Equation (13) has solution $y=\operatorname{dn}(p t)$, where $\operatorname{dn}$ is a Jacobian elliptic function with modulus $k$ (Whittaker and Watson, 1962). Thus:

$$
\sin \theta=\sin \alpha \operatorname{dn}(p t)
$$

The function $\operatorname{dn}(p t)$ oscillates between the values 1 and $k_{1}$, (where $k_{1}$ is the complementary modulus defined by $\left.k_{1}^{2}+k^{2}=1\right)$ and has time period $2 K / p$, where $K$ is the complete elliptic integral of the first kind (Bowman, 1953).

From equation (18) $\sin \theta$ varies between $\sin \alpha$ and $k_{1} \sin \alpha=\sin \beta$. It may be concluded that $\theta$ is periodic with time period $2 K / p$ and with bounding values $\alpha$ and $\beta$. This oscillation of the tilt angle $\theta$ is known as nutation.

Equation (11) shows that the twist rate has the $\operatorname{sign}$ of $h \sin \theta$ and so $\psi$ is monotonic.

Equations (8) and (9) show that $\theta=\alpha$ corresponds to the zero and half twist positions, while $\theta=\beta$ corresponds to the quarter and three-quarter twist positions so that the time taken for a full twist is twice the period of $\theta$. Thus the average twist rate is:

$$
n=\pi p / 2 K
$$

Equation (2) shows that the somersault rate $\dot{\phi}$ varies between $h / A$ and $h / B$ and equation (10) indicates that the time period of the oscillations is the same as the period of $\theta$, namely $2 K / p$.

From equation (10) the average somersault rate is:

$$
\Omega=h[1 / C-(1 / C-1 / A) V(\alpha)]
$$

where $V(\alpha)$ is the average value of $\cos ^{2} \alpha / \cos ^{2} \theta . \quad V(\alpha)$ may be expressed in terms of Jacobi's Zeta function and an elliptic integral of the first kind as shown in Appendix 1.

The general motion of a rigid body in the rod mode may now be described. The somersault angle $\phi$ and the twist angle $\psi$ steadily increase, while the tilt angle $\theta$ oscillates between $\alpha$ and $\beta$ (assuming that $h, \alpha, \beta>0)$.

At the half and full twist positions, $\sin \psi=0$ so that:

(3), (8): $\quad \theta$ attains its maximum value of $\alpha$ and $\dot{\theta}=0$

(2): $\quad \dot{\phi}$ attains its minimum value of $h / A$

(4): $\quad \dot{\psi}$ attains its maximum value of $(h / C-h / A) \sin \alpha$

At the quarter and three-quarter twist positions, $\cos \psi=0$ so that:

(3),(9): $\quad \theta$ attains its minimum value of $\beta$ and $\dot{\theta}=0$

(2): $\quad$ attains its maximum value of $h / B$

(4): $\quad$ attains its minimum value of $(h / C-h / B) \sin \beta$

During the motion, the average somersault and twist rates are given by equations (20) and (19).

The rod mode may be described as a twisting somersault. An example is shown later in Fig. 4. In future, this mode of motion will be referred to as the twisting mode. 


\section{The motion of a rod}

A rod has principal moments of inertia $A, B, C$ such that $A=B>C$. From equation (13), $\cos ^{2} \alpha_{0}=1$ so that $\alpha>\alpha_{0}$ providing $\theta \neq 0$, so that a rod must move in the twisting mode. From equations

$$
\begin{array}{ll}
(2): & \dot{\phi}=h / A \\
(3): & \dot{\theta}=0 \\
(4): & \dot{\psi}=(h / C-h / A) \sin \theta
\end{array}
$$

and so the somersault rate has the constant value $\Omega$ where:

$$
\Omega=h / A
$$

the tilt angle $\theta$ has the constant value $\alpha$, and the twist rate has the constant value $p$ where:

$$
p=\Omega(A / C-1) \sin \alpha
$$

In the Introduction, it was noted that equations were also provided by Eaves (1969), Eaves (1971)) and Frohlich (1979). There are discrepancies between the various equations and these may be explained as follows.

Figure 2 shows that the angular velocity vector $\boldsymbol{\omega}$ may be resolved into components $\omega_{1}$ and $\omega_{3}$ along the body axes $\mathbf{f}_{1}$ and $\mathbf{f}_{3}$ or may be resolved into components $\Omega$ and $p$ along axes $\mathbf{i}_{1}$ and $\mathbf{f}_{3}$ where $\mathbf{i}_{1}$ is fixed in space. It has been shown above that $\Omega$ and $p$ are the rates of change of the somersault and twist angles, and so $\Omega$ and $p$ may be referred to as the somersault and twist rates. On the other hand, $\omega_{1}$ and $\omega_{3}$ are components of the angular velocity along the principal axes $\mathbf{f}_{1}$ and $\mathbf{f}_{3}$ and while it is permissible to refer to them as the somersault and twist angular velocities, this can be misleading since $\Omega$ and $p$ are also angular velocities associated with somersault and twist.

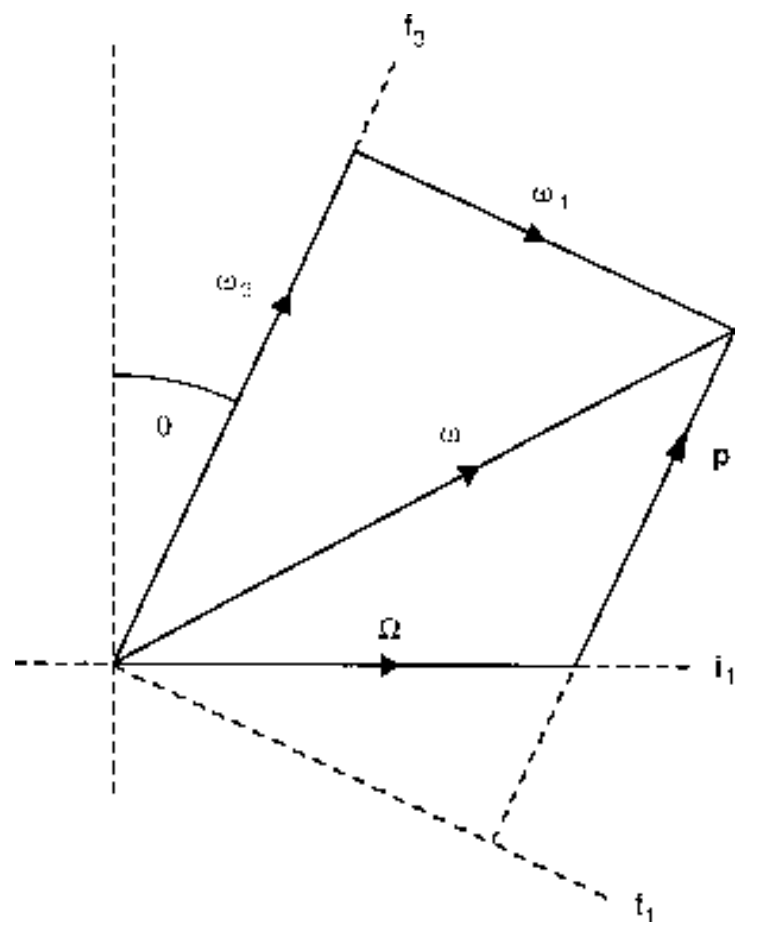

Figure 2: Decomposition of a rod's angular velocity $\boldsymbol{\omega}$ as $\omega_{1} \mathbf{f}_{\mathbf{1}}+\omega_{3} \mathbf{f}_{\mathbf{3}}$ and as $\Omega \mathbf{i}_{\mathbf{1}}+p \mathbf{f}_{\mathbf{3}}$.

Resolving along $\mathbf{f}_{1}$ and $\mathbf{f}_{3}$ gives:

$$
\begin{gathered}
\omega_{1}=\Omega \cos \theta \\
\omega_{3}=p+\Omega \sin \theta
\end{gathered}
$$


In Fig. 3 the angular momentum vector is parallel to $\mathbf{i}_{1}$ and has components $A \omega_{1}$ and $C \omega_{1}$, along the principal axes $\mathbf{f}_{1}$ and $\mathbf{f}_{3}$. Because of the symmetry equation $A=B$, the principal axis $\mathbf{f}_{1}$ may be chosen to lie in the plane defined by $\mathbf{i}_{1}$ and $\mathbf{f}_{3}$ and so the angular velocity $\omega_{2}=\dot{\theta}$ must be zero, since there is no component of angular momentum perpendicular to this plane. Thus the tilt angle $\theta$ has a constant value.

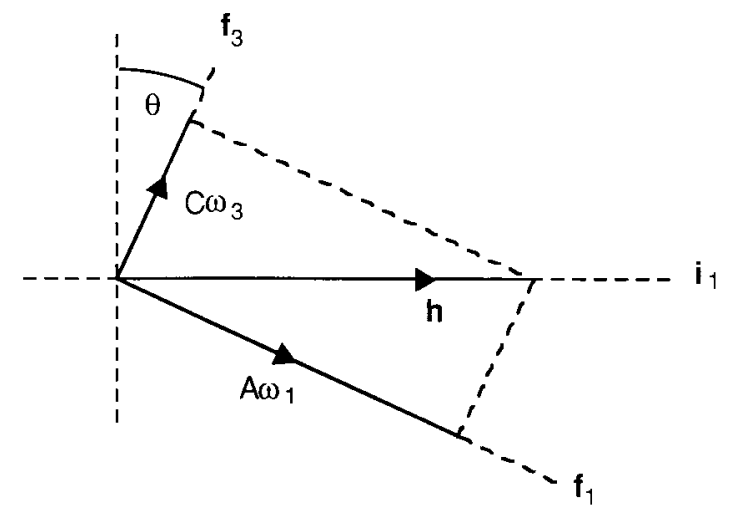

Figure 3: Decomposition of a rod's angular momentum $\mathbf{h}$ as $A \omega_{1}+C \omega_{3}$.

Resolving parallel and perpendicular to $\mathbf{i}_{1}$, gives:

$$
\begin{gathered}
h=A \omega_{1} \cos \theta+C \omega_{3} \sin \theta \\
C \omega_{3} \cos \theta=A \omega_{1} \sin \theta
\end{gathered}
$$

The last equation may be written as:

$$
\omega_{3}=\omega_{1}(A / C) \tan \theta
$$

and is equivalent to that used by Eaves (1969). Substituting for $\omega_{1}$ gives:

$$
\omega_{3}=\Omega(A / C) \sin \theta
$$

which is equivalent to that given by Frohlich (1979). Substituting for $\omega_{3}$ produces equation (25) again:

$$
p=\Omega(A / C-1) \sin \theta
$$

While each of equations (25), (26) and (27) describes the motion, only equation (25) gives the number of twists per somersault.

Eaves (1971) analysed the motion of a rod by considering the movement of the momental ellipsoid on the invariable plane (described in Synge and Griffith (1959)). Such an analysis leads once again to equation (25):

$$
p=\Omega(A / C-1) \sin \theta
$$

The momental ellipsoid has semi-axes inversely proportional to the square roots of the principal moments of inertia so that the ellipsoid is cigar-shaped. Eaves, however, took the semi-axes to be directly proportional to the principal inertias and deduced that the ellipsoid was disc-shaped. This error accounts for the discrepancy between equation (25) and the statement of Eaves that:

$$
p \approx \Omega \sin \theta
$$

\section{The wobbling mode}

If a disc is defined to be an axially symmetric body with principal moments of inertia $A, B, C$ such that $A>B=C$, then from equation (13) $\cos ^{2} \alpha_{0}=0$ so that $\alpha \leq \alpha_{0}$. For a general rigid body, the motion will be said to be in the disc mode providing $\alpha<\alpha_{0}$. From equation (14) this condition is equivalent 
to $c_{\beta}^{2}>1$, which together with equation (9) implies that $\cos \psi$ is never zero, so that the quarter twist position is not attained during the motion.

Equation (12) may be written as:

$$
\dot{y}^{2}=q^{2}\left(1-y^{2}\right)\left(k^{2} y^{2}+k_{1}^{2}\right)
$$

where: $q^{2}=h^{2} \cos ^{2} \alpha(A-C)(A-B) / A^{2} B C, k^{2}=\tan ^{2} \alpha A(B-C) / C(A-B)<1$ and $k_{1}^{2}=1-k^{2}$.

Equation (28) has solution $y=\mathrm{cn}(q t)$, where $\mathrm{cn}$ is a Jacobian elliptic function with modulus $k$ (Whittaker and Watson, 1962). Thus:

$$
\sin \theta=\sin \alpha \operatorname{cn}(q t)
$$

The function $\mathrm{cn}(q t)$ oscillates between 1 and -1 and has time period $4 K / q$, where $K$ is the complete elliptic integral of the first kind (Bowman, 1953) and may be evaluated using equation (15). As a consequence, $\theta$ is periodic with time period $4 K / q$ and with bounding values $\alpha$ and $-\alpha$. As in the twisting mode, the tilt angle $\theta$ oscillates so the minor axis $\mathbf{f}_{3}$ nutates.

Equation (11) shows that the twist rate $\dot{\psi}$ has the $\operatorname{sign}$ of $h \sin \theta$ and is zero only when $\theta=0$. Thus $\psi$ oscillates between $\psi_{0}$ and $-\psi_{0}$ where the angle $\psi_{0}$ corresponds to $\theta=0$ and is given by equation (8) as:

$$
\sin ^{2} \psi_{0}=\sin ^{2} \alpha B(A-C) / C(A-B)>\sin ^{2} \alpha
$$

The time period of $\psi$ is the same as the period of $\theta$, namely $4 K / q$, and since $\psi$ is oscillatory the average twist rate is zero.

Equation (10) shows that the somersault rate $\dot{\phi}$ varies between $h / A$ and $h \cos ^{2} \alpha / A+h \sin ^{2} \alpha / C$ and has time period one-half that of $\theta$, namely $2 K / q$. From equation (10) the average somersault rate is:

$$
\Omega=h[1 / C-(1 / C-1 / A) W(\alpha)]
$$

where $W(\alpha)$ is the average value of $\cos ^{2} \alpha / \cos ^{2} \theta$.

From equation (29) $\sin \theta=\sin \alpha \operatorname{cn}(q t)$ so that:

$$
\begin{aligned}
\cos ^{2} \theta & =1-\sin ^{2} \alpha \mathrm{cn}^{2} q t \\
& =1-\sin ^{2} \alpha\left(1-\operatorname{sn}^{2} q t\right) \\
& =\cos ^{2} \alpha+\sin ^{2} \operatorname{sn}^{2} u
\end{aligned}
$$

where $u=q t$. Thus:

$$
\begin{aligned}
W(\alpha) & =\frac{1}{K} \int_{0}^{K} \frac{d u}{1+\tan ^{2} \alpha \operatorname{sn}^{2} u}\left(\text { where } k \tan \alpha_{1}=\tan \alpha\right) \\
& =V\left(\alpha_{1}\right)
\end{aligned}
$$

where $V$ is given in Appendix 1

The general motion of a rigid body in the disc mode may now be described. The somersault angle $\phi$ steadily increases (for $h>0$ ), while the tilt angle $\theta$ oscillates between $\pm \alpha$ and the twist angle $\psi$ oscillates between $\pm \psi_{0}$.

As the time $t$ increases from 0 to the period $4 K / q$, the oscillations occur in sequence:

$$
\begin{array}{llll}
t=0 & \theta=\alpha & \psi=0 & \dot{\phi} \text { is a minimum } \\
t=K / q & \theta=0 & \psi & \dot{\phi} \text { is a maximum } \\
t=2 K / q & \theta=-\alpha & \psi & \dot{\phi} \text { is a minimum } \\
t=3 K / q & \theta=0 & \psi & \dot{\phi} \text { is a maximum }
\end{array}
$$

The average somersault rate is given by equation (30).

The disc mode may be described as a wobbling somersault. An example is shown later in Fig. 5. In future, this mode of motion will be referred to as the wobbling mode. 


\section{The motion of a disc}

A disc has principal moments of inertia $A, B, C$ such that $A>B=C$. For a disc $\cos ^{2} \alpha_{0}=0$ so that $\alpha<\alpha_{0}$ providing $\theta \neq 90^{\circ}$ so that a disc must move in the wobbling mode. With $B=C$, equation (28) becomes:

$$
\dot{y}^{2}=q^{2}\left(1-y^{2}\right)
$$

where $y=\sin \theta=\sin \alpha \cos q t$ and $\theta$ oscillates between $\pm a$ with time period $2 \pi / q$.

Substituting for $\sin \theta$ in equation (8) and setting $B=C$ produces $\tan \psi=\tan \alpha \sin q t$, so that $\psi$ oscillates between $\pm \alpha$ with period $2 \pi / q$.

The average somersault rate $\Omega$ is given by equation (30) where $W(\alpha)$ is the average value of $\cos ^{2} \alpha / \cos ^{2} \theta$, so that:

$$
W(\alpha)=\frac{2}{\pi} \int_{0}^{\pi / 2} \frac{d u}{1+\tan ^{2} \alpha \sin ^{2} u}
$$

which may be integrated using the substitution $\tan u=\cos \alpha \tan v$ to give $W(\alpha)=\cos (\alpha)$. Thus $\Omega=h[1 / C-(1 / C-1 / A) \cos \alpha]$ is the average somersault rate and the number of somersaults per oscillation is given by:

$$
\Omega / q=\sec \alpha[A /(A-C)]-1
$$

and is greater than 1 since $C \geq A / 2$.

\section{The singular solution separating the twisting and wobbling modes}

Two modes of motion have been identified corresponding to the cases (a) $\alpha>\alpha_{0}$ and (b) $\alpha<\alpha_{0}$ where $\alpha$ is the value of the tilt angle for zero twist and $\alpha_{0}$ is defined in terms of the principal moments of inertia by equation (13). The case (c) $\alpha=\alpha_{0}$ will be considered in this section as the solution separating the twisting mode and the wobbling mode.

For a rigid body with $A>B>C$, the condition $2 T=h^{2} / B$ is satisfied by steady rotations about the axis corresponding to the intermediate principal moment $B$. In addition, there is a motion in which rotation about the intermediate axis in one direction leads to a half twist followed by rotation in the opposite direction.

Equation (6) shows that the condition $2 T=h^{2} / B$ is equivalent to:

$$
\sin ^{2} \alpha_{0}=C(A-B) / B(A-C)
$$

where $\alpha_{0}$ is the angle of tilt when the twist angle $\psi$ is zero. This corresponds to the situation $\alpha=\alpha_{0}$. Equation (7) shows that $2 T=h^{2} / B$ is equivalent to:

$$
\beta_{0}=0
$$

where $\beta_{0}$ is the angle of tilt at the quarter twist position. Equation (12) becomes:

$$
\dot{y}^{2}=d^{2} y^{2}\left(1-y^{2}\right)
$$

where $d^{2}=h^{2}(A-B)(B-C) / A B^{2} C$.

Equation (32) has solution

$$
y=\operatorname{sech}(d t)=2 /(\exp (d t)+\exp (-d t))
$$

and so:

$$
\sin \theta=\sin \alpha_{0} \operatorname{sech}(d t)
$$

The function $\operatorname{sech}(d t)$ has a maximum value of 1 at $t=0$ and approaches zero for large values of $|t|$ and so $\theta=\alpha_{0}$ at $t=0$ with $\theta$ decaying to zero as $|t|$ approaches infinity.

Equations (9) and (33) lead to:

$$
\tan ^{2} \psi=\sec ^{2} \alpha_{0} \sinh ^{2} d t
$$

and since equation (11) implies that $\dot{\psi}$ has the $\operatorname{sign}$ of $h \sin \theta$, the twist angle $\psi$ is given by:

$$
\tan \psi=\sec \alpha_{0} \sinh (d t)
$$


in the case $h \alpha_{0}>0$. Thus the twist angle increases from $90^{\circ}$ at $t=-\infty$, through zero at $t=0$, to $+90^{\circ}$ at $t=+\infty$.

With $\alpha=\alpha_{0}$ equation (10) may be rewritten as:

$$
\dot{\phi}=h\left[1 / B-(1 / C-1 / B) \tan ^{2} \theta\right]
$$

which gives the somersault rate as a function of time using equation (33). The resulting expression for $\dot{\phi}$ may be integrated to give:

$$
\phi=(h / B) t-\arctan \left[\tan \alpha_{0} \tanh (d t)\right]
$$

where:

$\tanh (d t)=[\exp (d t)-\exp (-d t)] /[\exp (d t)+\exp (-d t)]$

The somersault rate $\dot{\phi}$ decreases from $h / B$ at $t=-\infty$ to $h / A$ at $t=0$ and then increases to $h / B$ as $t$ approaches $+\infty$. Since the time period of the oscillation is infinite and for large values of $|t|$ equation (35) gives the somersault angle as approximately $(h / B) t-\alpha_{0} \operatorname{sign}(t)$, the average somersault rate is equal to $h / B$ for an infinite number of somersaults.

Equation (11) shows that the twist rate has a maximum value of $h(1 / C-1 / A) \sin \alpha_{0}$ at time $t=0$ and approaches zero for large values of $|t|$. Over the infinite time period of the half twist the average twist rate is zero, although at any instant the actual twist rate is greater than zero.

At time $t=0$ the ratio $\dot{\psi} / \dot{\phi}$ has the maximum value:

$$
\begin{aligned}
\max [\dot{\psi} / \dot{\phi}] & =\left[\frac{A}{C}-1\right] \sin \alpha_{0} \\
& =\left[\frac{A}{C}-1\right]^{1 / 2}\left[\frac{A}{B}-1\right]^{1 / 2}
\end{aligned}
$$

In this idealized case in which $2 T=h^{2} / B$, the motion of a rigid body comprises an infinite number of forward somersaults (for $h>0$ ), followed by a finite number of somersaults in which the half twist becomes evident, followed by an infinite number of backward somersaults. In reality, $2 T$ will never be exactly equal to $h^{2} / B$ and the motion will be either in the wobbling mode or the twisting mode and the oscillation period of $\theta$ will be finite. An example is shown later in Fig. 6. Such motions for which $2 T$ is approximately equal to $h^{2} / B$ will have the following characteristics which are not dependent upon the mode of motion.

During the oscillation of the tilt angle $\theta$, the motion will exhibit the following three phases:

(a) somersault in one direction with no apparent twist;

(b) somersault during which a half twist occurs; and

(c) somersault in the opposite direction with no apparent twist.

This cycle of movement will be repeated throughout the motion and in the twisting mode the twist is a monotonic function of time so that consecutive half twists will be in the same direction, while in the wobbling mode the twist is oscillatory so that consecutive twists will be in opposing directions. In the wobbling mode, the amount of twist in a cycle will be slightly less than a half twist.

If a rigid body is initially rotating about an axis close to the intermediate principal axis, $\alpha$ will be approximately equal to $\alpha_{0}$ and the subsequent motion will consist of a sequence of half twists in which the twist rate varies from a value close to zero to a maximum at the quarter twist position. In other words, rotations about the intermediate principal axis are unstable.

The above discussion serves three purposes:

(a) It shows how the twisting and wobbling modes approach each other;

(b) it explains the instability of rotations about the intermediate principal axis; and

(c) it provides a description of the motion in such cases. 


\section{Simulations}

In order to illustrate the different modes of motion, a simulation model has been used (Yeadon et al., 1990). Values for the principal moments of inertia have been calculated from anthropometric measurements of the 1982 Men's World Trampoline Champion using an inertia model (Yeadon, 1990b). The body reference axes of the simulation model lie within $2^{\circ}$ of the principal axes of inertia in each configuration used. In each of the following three simulations, a fixed configuration has been maintained so that the system behaves as a single rigid body. The characteristics of each simulation have also been derived independently using equations (1)-(3).

Each configuration is symmetrical about the sagittal plane so that the lateral axis $\mathbf{f}_{1}$ will be principal with principal moment of inertia $I_{1}$. If $A, B, C$ are the principal moments of inertia with $A>B>C$, then:

(a) $I_{1}=A$ when the pike angle is less than $137^{\circ}$; and

(b) $I_{1}=B$ when the pike angle is greater than $137^{\circ}$.

In case (a) the reference frame $f$ used in the development of the rigid body equations is the same as that of the simulation model, whereas in case (b) there is a $90^{\circ}$ phase difference between the two twist angles. Thus in case (b) the rigid body equations should be modified by changing $\psi$ to $\psi-90^{\circ}$.

For convenience, the unit of time is taken to be the duration of the simulation and the simulation program uses normalized angular momentum values as input data with a value of 1 corresponding to the momentum of a straight somersault.

\section{The twisting somersault simulation RD1}

During the simulation RD1, the body maintains a straight position and the principal moments of inertia $A, B, C$ are:

$$
A=11.01 \quad B=10.56 \quad C=0.70 \quad\left(\mathrm{~kg} \cdot \mathrm{m}^{2}\right)
$$

The initial tilt angle is $\beta=10^{\circ}$ and equation (16) gives:

$$
(1 / C-1 / A) \cos ^{2} \alpha=(1 / C-1 / B) \cos ^{2} \beta
$$

from which $\alpha=10.5^{\circ}$ and the tilt angle $\theta$ oscillates between these two values during the simulation.

From equation (2) the somersault rate varies between $h / B$ and $h / A$ and in this simulation the angular momentum has a normalized value of 1 so that $h / B$ corresponds to a somersault rate of 1 revolution per unit time. Thus the somersault rate varies between 1.00 and 0.96 revolutions per unit time (Fig. 4).

The twist rate is given by equation (4) as:

$$
\dot{\psi}=(h / C-\dot{\phi}) \sin \theta
$$

which varies between $(h / C-h / B) \sin \beta$ and $(h / C-h / A) \sin \alpha$. Thus the twist rate varies between 2.46 and 2.58 revolutions per unit time (Fig. 4).

Equation (25) may be used to obtain the average number of twists per somersault in the form:

$$
p / \Omega=(I / C-1) \sin \theta
$$

Taking $I$ to be the average of $A$ and $B$, and $\theta$ to be the average of $\alpha$ and $\beta$, gives $p / \Omega=2.57$ twists per somersault, which is in agreement with the value obtained from the simulation.

The motion may be described as a forward somersault with $2 \frac{1}{2}$ twists (Fig. 4).

\section{The wobbling somersault simulation DS1}

During the simulation DS1, the body maintains a $60^{\circ}$ angle of pike and the principal moments of inertia $A, B, C$ are:

$$
A=5.78 \quad B=3.83 \quad C=3.10 \quad\left(\mathrm{~kg} \cdot \mathrm{m}^{2}\right)
$$

Initially, $\psi=0$ and the angle of tilt is $\alpha=10^{\circ}$ and so the tilt angle oscillates between $-10^{\circ}$ and $+10^{\circ}$. 


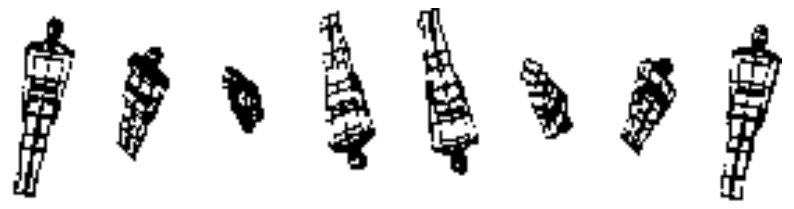

\section{twist}

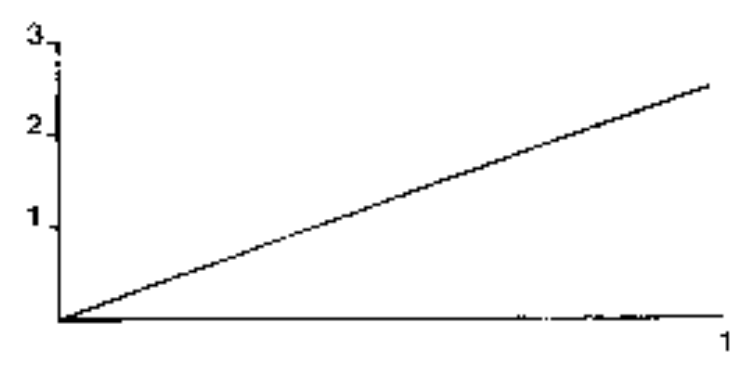

somersault

Figure 4: Oscillation during the twisting somersault simulation RD1.
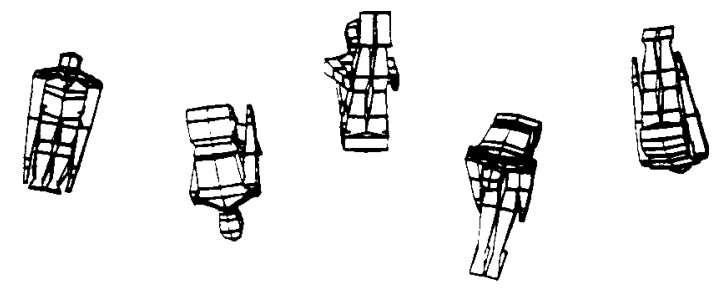

\section{twist}

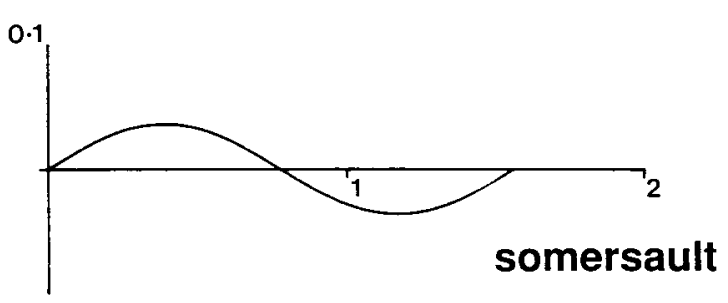

Figure 5: Oscillation during the wobbling somersault simulation DS1.

At $\theta=0$ equation (8) gives the twist angle $\psi_{0}$ as:

$$
\sin ^{2} \psi_{0}=\sin ^{2} \alpha B(A-C) / C(A-B)
$$

so that $\psi_{0}=13.1^{\circ}$ and the twist angle oscillates between $-13.1^{\circ}$ and $+13.1^{\circ}$ (Fig. 5).

Equation (10) shows that the somersault rate varies from $h / A$ to $h\left[1 / C-(1 / C-1 / A) \cos ^{2} \alpha\right]$, which are in the ratio 1:1.026 and the disc equation:

$$
\Omega=h[1 / C-(1 / C-1 / A) \cos \alpha]
$$


may be used to give the average somersault rate as $(1.013) h / A$.

In equation (28) the modulus $k$ is given by $k^{2}=\tan ^{\alpha} A(B-C) / C(A-B)$ so that $k=0.147$, the complementary modulus $k_{1}=0.989$ and $K=1.579$.

The time period of an oscillation is $4 K / q$, where $q$ is obtained from: $q^{2}=h^{2} \cos ^{2} \alpha(A-C)(A-$ $B) / A^{2} B C$ as $(0.653) h / A$. The number of somersaults per oscillation can then be calculated as $2 k \Omega / \pi q=$ 1.56 , which is in close agreement with the simulation value.

Figure 5 shows the orientation of the body at quarter cycle intervals. The motion may be described as a wobbling somersault.

\section{The singular solution}

In the simulation SN1, the body maintains a straight position and the principal moments of inertia $A, B, C$ are:

$$
A=11.01 \quad B=10.56 \quad C=0.70 \quad\left(\mathrm{~kg} \cdot \mathrm{m}^{2}\right)
$$

In the singular solution, the tilt angle at the quarter twist position is $\alpha_{0}$, where:

$$
\sin ^{2} \alpha_{0}=C(A-B) / B(A-C)
$$

so that $\alpha_{0}=3.1^{\circ}$. During the motion, the tilt angle increases from 0 to $3.1^{\circ}$ and then decreases to zero again. The somersault rate decreases from $h / B$ to $h / A$ and then increases to $h / B$ again (Fig. 6).
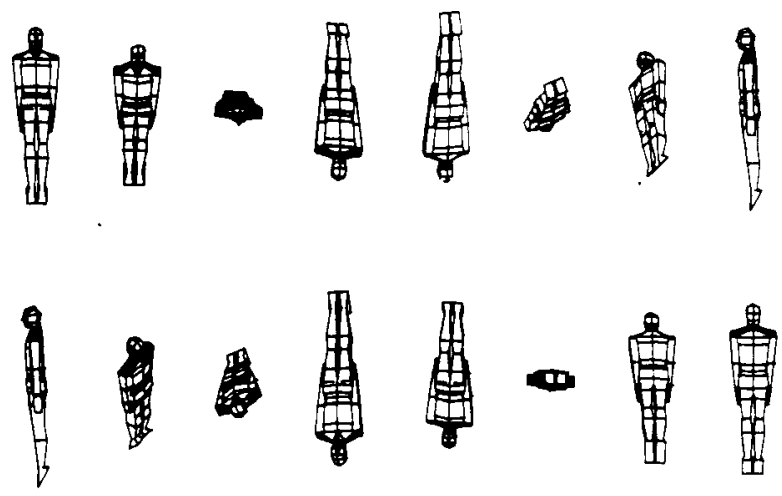

\section{twist}

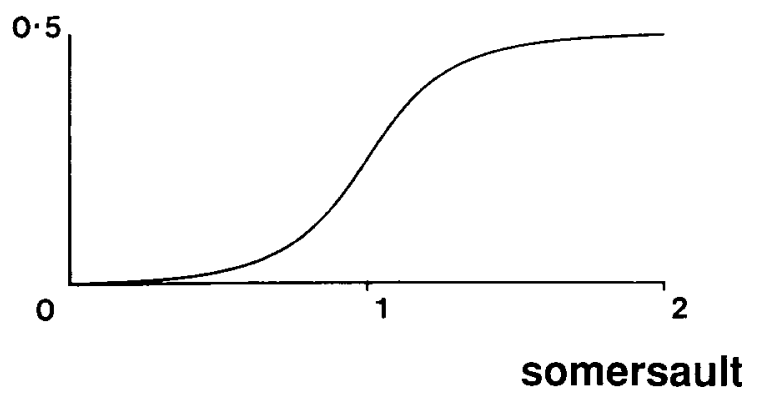

Figure 6: Oscillation during the singular solution SN1.

Equation (36) gives the maximum of the ratio $\dot{\psi} / \dot{\phi}$ as:

$$
\begin{aligned}
\max [\dot{\psi} / \dot{\phi}] & =\left[\frac{A}{C}-1\right] \sin \alpha_{0} \\
& =\left[\frac{A}{C}-1\right]^{1 / 2}\left[\frac{A}{B}-1\right]^{1 / 2}
\end{aligned}
$$


which is about 0.8 twists per somersault.

In the singular solution, the time period of the oscillation is infinite, whereas the simulation SN1 was created with initial conditions $\theta=0^{\circ}, \psi_{0}=0.015^{\circ}$, which resulted in two somersaults for the oscillation. The motion SN1 is in fact in the wobbling mode with the twist angle increasing from $\psi_{0}$ to $\left(180-\psi_{0}\right)$ during the two somersaults. The majority of the twist takes place between $\frac{3}{4}$ and $1 \frac{1}{4}$ somersaults. As a result, the motion appears to start as a non-twisting forward somersault and end as a non-twisting backward somersault (Fig. 6).

\section{Instability}

Since the twisting mode and wobbling mode are quite different modes of motion, it is of interest to see how they approach each other when $\alpha$ is close to $\alpha_{0}$. Figure 7 shows how the twist angle varies with the somersault angle in each case. In each mode, the twist rate varies from 0 to 0.8 twists per somersault and each movement may be described as a quadruple somersault with two distinct half twists. The difference is that in the twisting mode these half twists are in the same direction, whereas in the wobbling mode the half twists are in opposite directions.

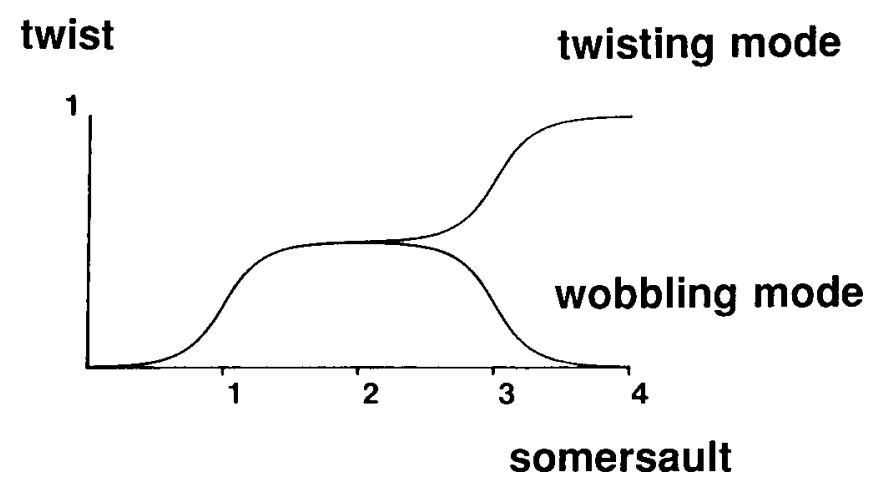

Figure 7: Twisting mode and wobbling mode solutions which approach each other.

If the body is initially rotating about an axis which is close to the intermediate principal axis, then the initial tilt angle $\beta$ will be close to zero. The tilt angle will rise to $\alpha$ (approximately $\alpha_{0}$ ) at the quarter twist position and the motion will resemble one of the modes shown in Fig. 7. This discussion not only establishes the instability of such rotations, as has already been demonstrated by Nigg (1974) and Hinrichs (1978), but also describes the subsequent motion.

It might be thought that abducting the arms in layout somersaults will reduce this instability. The preceding analysis shows that this is not so and equation (36) shows that the maximum value of the twist rate will be greater in a somersault with abducted arms. If a fixed configuration is to be maintained, the only possibility is to pike or arch sufficiently so as to make the lateral axis the maximum principal axis rather than the intermediate principal axis. If variable body configurations are permitted, there exists the possibility of making inflight adjustments to keep the tilt angle and twist angle close to zero. An analysis of such a control mechanism is given by Yeadon and Mikulcik (1991).

\section{Discussion}

It has been shown that the torque-free rotational motions of a rigid body fall into two general classes - the twisting mode and the wobbling mode. In the twisting mode the twist angle steadily increases, whereas in the wobbling mode the twist angle oscillates about a mean value with amplitude of less than a quarter twist. This suggests that it may be possible to change from one mode to the other by means of suitable configurational changes during flight. Such configurational changes may induce a change of mode via the inertia parameter values or by changing the tilt angle. The effect of moving from one mode to the other will be to change a non-twisting somersault into a twisting somersault or vice versa. In other words, a change of mode has relevance to the initiation and termination of twist. 
Relationships between the angles of somersault, tilt and twist and their rates of change have been determined and the conflict between the results of Eaves (1969), Eaves (1971) and Frohlich (1979) thereby resolved. These equations describe how the tilt angle changes in the twisting and wobbling modes and suggest how such nutation may be used to advantage. In the twisting mode, for example, the tilt angle will reach a maximum at the quarter twist position. It will therefore be an advantage to wait until the quarter twist position is reached before reducing the moment of inertia about the longitudinal axis to accelerate the twist. In the wobbling mode, the tilt angle oscillates between positive and negative values and as the sign of the tilt angle changes so does the direction of twist. By remaining in the piked position until the tilt angle is close to zero, it should be possible to extend from the pike without a twisting velocity.

The two distinct modes of motion approach each other near a singular solution in which the nutation of the tilt angle is extreme. Near this solution the twist rate varies dramatically so that the twist appears to stop and restart. The reason that rotations about the minimum axis of inertia are stable is that such motions are central in the twisting somersault solution space and a small perturbation merely moves the trajectory into a similar twisting somersault with a tilt angle close to $90^{\circ}$. Rotations about the maximum moment of inertia are central in the wobbling somersault solution space and a small perturbation moves the trajectory into a similar somersault with a small amount of wobble. In contrast, rotations about the intermediate principal axis lie on the edge of both modes of motion, which are characterized as somersaults with periodic but distinct half twists in this region. Thus any small perturbation will move a non-twisting somersault about the intermediate axis into an extreme example of either a twisting somersault or a wobbling somersault. The instability will be manifested as a sudden half twist. Thus the instability of such rotations arises as a consequence of there having to be a transition between the two modes of motion which are distinct in nature and therefore take on extreme forms in order to approach each other along the singular solution. It may be that this phenomenon may be used to advantage in order to produce a twisting somersault with little effort.

The quantitative descriptions of rigid body motions will be applied to both contact twist (in Part II) and aerial twist (in Part III) and will provide the basis for the partitioning of performance into different twisting techniques (in Part IV). A knowledge of all possible motions of a rigid body will permit the identification of fundamental effects such as nutation in more complex movements and this will lead to insights into the operation of twisting techniques.

\section{References}

Aaron, G. S. (1977). The Science of Trampolining. Muller, London.

Batterman, C. (1974). The Techniques of Springboard Diving. MIT Press, Cambridge, Mass.

Bowman, F. (1953). Introduction to Elliptic Functions with Applications. English Universities Press, London.

Eaves, G. (1960). The falling cat and the twisting diver. New Scientist, 8:249.

Eaves, G. (1969). Diving: The Mechanics of Springboard and Firmboard Techniques. Kaye and Ward, London.

Eaves, G. (1971). Recent developments in the theory of twist dives. In Lewillie, L. and Clarys, J. P., editors, First International Symposium on Biomechanics in Swimming, Waterpolo and Diving, pages $237-242$.

Frohlich, C. (1979). Do springboard divers violate angular momentum conservation? American Journal of Physics, 47:583-592.

Frohlich, C. (1980). The physics of somersaulting and twisting. Scientific American, 242:112-120.

Hinrichs, R. N. (1978). Principal axes and moments of inertia of the human body: An investigation of the stability of rotary motions. Master's thesis, University of Iowa. Unpublished.

McDonald, D. (1960). How does a cat fall on its feet? New Scientist, 7:1647-1649. 
Nigg, B. M. (1974). Analysis of twisting and turning movements. In Nelson, R. C. and Morehouse, C. A., editors, Biomechanics IV, pages 279-283. University Park Press, Baltimore.

Pike, N. L. (1980). Computer simulation of a forward, full twisting dive in a layout position. PhD thesis, Pennsylvania State University.

Rackham, G. (1960). The origin of twist. Swimming Times, 37:116-118.

Smith, G. (1980). Basic twisting concepts for gymnastics. International Gymnast, 22:TS10-TS13.

Synge, J. and Griffith, B. (1959). Principles of Mechanics. McGraw-Hill, London.

Whittaker, E. T. (1937). A Treatise on the Analytical Dynamics of Particles and Rigid Bodies. Cambridge University Press, Cambridge.

Whittaker, E. T. and Watson, G. N. (1962). A Course of Modern Analysis. Cambridge University Press, Cambridge.

Yeadon, M. R. (1990a). The simulation of aerial movement - I. The determination of orientation angles from film data. Journal of Biomechanics, 23:59-66.

Yeadon, M. R. (1990b). The simulation of aerial movement - II. A mathematical inertia model of the human body. Journal of Biomechanics, 23:67-74.

Yeadon, M. R., Atha, J., and Hales, F. D. (1990). The simulation of aerial movement - IV. A computer simulation model. Journal of Biomechanics, 23:85-89.

Yeadon, M. R. and Mikulcik, E. C. (1991). Control strategies for non-twisting somersaults. In Communication to the XIIIth International Society of Biomechanics Congress. Perth.

\section{Appendix 1: Evaluation of $V(\alpha)$}

$V(\alpha)$ is the average value of $\cos ^{2} \alpha / \cos ^{2} \theta$. From equation (18) $\sin \theta=\sin \alpha \operatorname{dn}(p t)$ so that:

$$
\begin{aligned}
\cos ^{2} \theta & =1-\sin ^{2} \alpha \mathrm{dn}^{2} p t \\
& =1-\sin ^{2} \alpha\left(1-k^{2} \operatorname{sn}^{2} p t\right) \\
& =\cos ^{2} \alpha+k^{2} \sin ^{2} \alpha \operatorname{sn}^{2} u
\end{aligned}
$$

where $u=p t$. Thus:

$$
V(\alpha)=\frac{1}{K} \int_{0}^{K} \frac{d u}{1+k^{2} \tan ^{2} \alpha \operatorname{sn}^{2} u}
$$

which is Legendre's complete elliptic integral of the third kind.

Writing $\tan ^{2} \alpha=-\operatorname{sn}^{2} a$ leads to:

$$
\begin{gathered}
V(\alpha)=\frac{1}{K}\left[K+\frac{\operatorname{sn} a}{\operatorname{cn} a \cdot \operatorname{dn} a} \pi(K, a)\right] \\
\text { where } \pi(K, a)=\int_{0}^{K} \frac{k^{2} \operatorname{sn} a \cdot \operatorname{cn} a \cdot \operatorname{dn} a \cdot \operatorname{sn}^{2} u \cdot d u}{1-k^{2} \operatorname{sn}^{2} a \operatorname{sn}^{2} u}
\end{gathered}
$$

is Jacobi's complete elliptic integral of the third kind and is equal to $K Z(a)$, where $Z$ is Jacobi's Zeta function (Whittaker and Watson, 1962) so that:

$$
V(\alpha)=1+\frac{\operatorname{sn} a}{\operatorname{cn} a \cdot \operatorname{dn} a} Z(a)
$$

Using Jacobi's imaginary transformation $a=i b$ (Whittaker and Watson, 1962) leads to:

$$
V(\alpha)=1-\operatorname{sn}_{1}^{2} b+\frac{\operatorname{sn}_{1} b \cdot \mathrm{cn}_{1} b}{\operatorname{dn}_{1} b}\left[Z_{1}(b)+\frac{\pi b}{2 K K_{1}}\right]
$$


where the function subscript 1 denotes that the modulus is $k_{1}$. Since

$$
\tan ^{2} \alpha=-\mathrm{sn}^{2} a=-\mathrm{sn}_{1}^{2} b=\operatorname{sn}_{1}^{2} b / \mathrm{cn}_{1}^{2} b
$$

and

$$
\sin ^{2} \alpha+\cos ^{2} \alpha=1=\operatorname{sn}_{1}^{2} b+\operatorname{cn}_{1}^{2} b
$$

it may be concluded that $\sin \alpha=\operatorname{sn}_{1} b$ so that $b=F_{1}(\alpha)$, where $F_{1}$ is an elliptic integral of the first kind with modulus $k_{1}$ and $V(\alpha)$ takes the form:

$$
\begin{aligned}
V(\alpha)= & \cos ^{2} \alpha+\sin \alpha \cos \alpha\left(1-k_{1}^{2} \sin ^{2} \alpha\right)^{-1 / 2}\left[Z_{1}\left(F_{1}(\alpha)\right)\right. \\
& \left.+\pi F_{1}(\alpha) / 2 K K_{1}\right]
\end{aligned}
$$

